\title{
CONCERNING THE UNIFORMIZATION OF CERTAIN RIEMANN SURFACES ALLIED TO THE INVERSE- COSINE AND INVERSE-GAMMA SURFACES
}

\author{
BY \\ GERALD R. MACLANE
}

1. Introduction $\left({ }^{1}\right)$. The object of this paper is to consider the three classes of open simply-connected symmetric Riemann surfaces which result from the surfaces defined by the entire functions $w=\cos z^{1 / 2}, w=\cos z$, and $w=1 / \Gamma(z)$, when the branch points are displaced in an arbitrary fashion along the real axis. For each class it is proved that all members are parabolic and a representation of the corresponding entire function is obtained, which is precise in the sense that any such entire function maps the punched plane on to a surface of the class in question.

The method employed is approximation by elliptic surfaces. This avoids restrictive assumptions on the location of the branch points such as those involved in a line-complex representation.

In the terminology of Iversen [1, pp. 38-52] $\left({ }^{2}\right)$, certain members of each class of surfaces exhibit indirectly critical singularities, as well as either variety of directly critical singularity. To the best of the author's knowledge, all classes of surfaces in the literature which have been proved parabolic possess only directly critical singularities of the first kind, with the exception of the surfaces treated by F. E. Ulrich [1] which possess two directiy critical singularities, one of the first, and one of the second kind.

2. Description of surfaces. The symmetric semi-cosinic surface $\mathcal{F}$ covering the $w$-plane is determined by the sequence of real numbers $a_{k}(k=1,2, \cdots)$, with $a_{1}>0, a_{2 n \pm 1}>a_{2 n}$. $\mathcal{F}$ is composed of the sheets $S_{1}, S_{2}, \cdots, S_{k}, \cdots ; S_{1}$ is a replica of the $w$-plane cut along the positive real axis from $w=a_{1}$ to $w=\infty$; $S_{k}(k>1)$ is a replica of the $w$-plane cut along the real axis except for the interval between $a_{k-1}$ and $a_{k} . S_{1}$ and $S_{2}$ are joined along their cuts from $a_{1}$ to $+\infty$, forming a first order branch point over $w=a_{1} ; S_{k}$ and $S_{k+1}$ are joined along their cuts from $a_{k}$ to $(-)^{k-1} \infty$.

In the particular case $a_{k}=(-)^{k-1}, \mathcal{F}$ is the Riemann surface of the function $z=(\operatorname{arc} \cos w)^{2}$. Any symmetric semi-cosinic surface is topologically equivalent to this prototype, and therefore open and simply-connected.

The possible singularities of $\mathcal{F}$ may be classified as follows:

(1) $\left|a_{k}\right|<M(k=1,2, \cdots)$ and $\lim _{k \rightarrow \infty} a_{k}$ does not exist. Then $\mathcal{F}$ has a

Presented to the Society, August 22, 1946; received by the editors April 15, 1946.

(1) The author wishes to thank Professor F. E. Ulrich for arousing the writer's interest in the type problem and for his helpful criticisms.

(2) Numbers in brackets refer to the references listed at the end of the paper. 
single logarithmic branch point over $w=\infty$, that is, one directly critical singularity of the first kind.

(2) The sequence $a_{k}$ is unbounded. Then $\mathcal{F}$ has one directly critical singularity of the second kind over $w=\infty$.

(3) $\operatorname{Lim}_{k \rightarrow \infty} a_{k}=a \neq \infty$. Then $\mathcal{F}$ has two singularities: one directly critical of the first kind over $w=\infty$, the other directly critical over $w=a$.

The symmetric cosinic surface $\mathcal{F}$, covering the $w$-plane, bears the same relation to that of $z=\operatorname{arc} \cos w$ as the semi-cosinic surface bears to the surface of $z=(\operatorname{arc} \cos w)^{2} . \mathcal{F}$ consists of the sheets $S_{k}(k=0, \pm 1, \pm 2, \cdots) ; S_{k}$ is a replica of the $w$-plane slit along the real axis except for the segment from $a_{k}$ to $a_{k+1} ; S_{k}$ and $S_{k+1}$ are joined along their slits extending from $a_{k+1} . \mathcal{F}$ is determined by the sequence of real numbers $a_{k}(k=0, \pm 1, \pm 2, \ldots), a_{2 k \pm 1}>a_{2 k}$. We shall assume $a_{1}>0, a_{0}<0$, so that $w=0$ is in the unslit portion of $S_{0}$.

The symmetric gammic surface $\mathcal{F}$ is obtained by adding a pair of logarithmic ends to the initial sheet of the semi-cosinic surface. Let the sequence $a_{k}$ and the sheets $S_{k}$ be as described for the semi-cosinic surface. The sheet $S_{1}$ is opened up along the negative real axis from $-\infty$ to $-a_{0}, a_{0}>0$, and a logarithmic end spiraling over $\infty$ and $-a_{0}$ is attached to each shore of this cut. For later purposes it is convenient to break these up into half sheets. The end attached to the upper shore is built up of the half sheets $Q_{1}, Q_{2}, \ldots ; Q_{2 k-1}$ is a replica of $\Im w<0, Q_{2 k}$ of $\Im w>0 . Q_{1}$ is joined to the upper shore of $S_{1}$ along $\left(-\infty,-a_{0}\right), Q_{2 k-1}$ and $Q_{2 k}$ are joined on $\left(-a_{0}, \infty\right), Q_{2 k}$ and $Q_{2 k+1}$ on $\left(-\infty,-a_{0}\right)$. The other logarithmic end is similarly built up of half sheets $Q_{-1}, Q_{-2}, \cdots ; Q_{-(2 k+1)}$ being an image of $\Im w>0, Q_{-2 k}$ an image of $\Im w<0$, and so on.

This class of surfaces is based on the Riemann surface defined by the entire function $w=1 / \Gamma(-z)$ (cf. Lense [1], Ginzel [1]). In this prototype surface the logarithmic branch point lies over $w=0$ rather than $-a_{0}$; the algebraic branch points straddle out in a uniform fashion: $a_{n} \sim(-)^{n-1} \Gamma\left(n-h_{n}\right) / \log n$, $\lim h_{n}=0$. The type of the symmetric gammic surface has been considered by $F$. E. Ulrich [1] who proves, using Ahlfors' metric condition, that $\mathcal{F}$ is parabolic provided $\operatorname{sgn} a_{n}=(-)^{n-1}$ and $\left|a_{n}\right| \log n>m \exp (n / \log n)$ for some positive constant $m$.

The possible singularities of the cosinic and gammic surfaces may be classified in terms of the sequence $a_{k}$ as was done for the semi-cosinic surfaces.

We now proceed to treat the class of symmetric gammic surfaces in detail. The procedure for the other two classes is closely parallel and is therefore omitted.

3. Proof that all symmetric gammic surfaces are parabolic. Let $\mathcal{F}$, a symmetric gammic surface, be mapped onto the disc $|\zeta|<R \leqq \infty$ by the normalized function

$$
\zeta=\phi(w), \quad w=f(\zeta),
$$




$$
f(0)=0 \in S_{1}, \quad f^{\prime}(0)=1 .
$$

Let $\mathcal{F}$ be cut in to two hyperbolic surfaces by slicing $S_{1}$ from $-a_{0}$ to $a_{1}$ along the real axis, and $S_{k}$ from $a_{k-1}$ to $a_{k}$. Let $\mathcal{F}^{+}$be the part containing the upper half of $S_{1}, \mathcal{F}$ - the other. There is then an appropriate semi-disc $D:|\zeta|<R_{1}$ $\leqq \infty, J \zeta>0$, such that $\mathcal{F}^{+}$is the image of $D$ by a holomorphic function $w=f_{1}(\zeta)$ and such that the image of $-R_{1}<\zeta<R_{1}$ is the system of cuts separating $\mathcal{F}^{+}$ and $\mathcal{F}^{-}$. Using the Schwarz reflection principle, $f_{1}(\zeta)$ is holomorphic in the whole disc $|\zeta|<R_{1}$, which it maps onto $\mathcal{F}$. If $f_{1}(\zeta)$ is normalized we obtain (1). Thus the image of the branch point of $\mathcal{F}$ over $w=a_{n}$ is a point $\zeta=b_{n}$ of the real axis :

$$
f\left(b_{n}\right)=a_{n}, \quad 0<b_{1}<b_{2}<\cdots<b_{k} \rightarrow R .
$$

The zeros of $f^{\prime}(\zeta)$ are all simple and occur at the points $\zeta=b_{n}$.

The fundamental regions in the $\zeta$-plane, images of the sheets of $\mathcal{F}$, will be as follows: $S_{k}(k>1)$ is mapped onto a portion of $|\zeta|<R$ bounded by two curves $C_{k-1}$ and $C_{k}$ symmetric about the real axis and intersecting the real axis at $b_{k-1}$ and $b_{k}$ respectively. The segment $\left(a_{k-1}, a_{k}\right)$ of $S_{k}$ corresponds to the interval $\left(b_{k-1}, b_{k}\right)$; the two shores of the cut in $S_{k}$ commencing at $a_{k-1}$ correspond to the two symmetric halves of $C_{k-1}$, and the two shores of the cut from $a_{k}$ correspond to the two halves of $C_{k}$. $S_{1}$ is mapped into a part of $|\zeta|<R$ containing $\zeta=0$ and bounded by $C_{1}, \Gamma_{1}$, and $\Gamma_{-1}, \Gamma_{1}$ lying in the upper half circle and to the left of $C_{1}, \Gamma_{-1}$ being its reflection in the real axis. The interval $-R<\zeta<b_{1}$ corresponds to the interval $-a_{0}<w<a_{1}$ in $S_{1}, \Gamma_{1}$ to the upper shore of the cut $\left(-\infty,-a_{0}\right)$ in $S_{1}$, and $\Gamma_{-1}$ to the lower shore. The image of $Q_{n}(n=1,2, \cdots)$ is bounded by two curves $\Gamma_{n}$ and $\Gamma_{n+1}$, the image of $Q_{-n}$ by curves $\Gamma_{-n}, \Gamma_{-n-1}$ which are the reflections of $\Gamma_{n}$ and $\Gamma_{n+1}$ in the real axis. $\Gamma_{n}$ separates $\Gamma_{n+1}$ and $C_{1}$. Each of the curves $C_{k}$ and $\Gamma_{ \pm n}$ is a Querschnitt of the disc $|\zeta|<R$ and no two of these have points in common. These curves together with the interval $-R<\zeta<R$ of the real axis constitute the real paths of $f(\zeta)$, the locus of points for which $f(\zeta)$ is real. Aside from the symmetry, this description is topological, and indifferent to $R<\infty$ or $R=\infty$.

We now consider the elliptic approximating surface $\mathcal{F}_{n}$ which is assembled from the $n+1$ sheets $S_{1}, S_{2}, \ldots, S_{n}, S_{n+1}^{\prime}$ and the $2 n$ half sheets $Q_{ \pm 1}, Q_{ \pm 2}, \cdots, Q_{ \pm(n-1)}, Q_{ \pm n}^{\prime}$. The unprimed sheets are as in $\mathcal{F} ; S_{n+1}^{\prime}$ is obtained by closing $S_{n+1}$ smoothly across the cut $\left(a_{n+1},(-)^{n} \infty\right) ; Q_{n}^{\prime}$ and $Q_{-n}^{\prime}$ dissected are the same as $Q_{n}$ and $Q_{-n}$, but $Q_{n}^{\prime}$ and $Q_{-n}^{\prime}$ are now connected along their free shores rather than passing on to $Q_{n+1}$ and $Q_{-(n+1)}$.

Thus $\mathcal{F}_{n}$ is a $2 n+1$ sheeted simply-connected closed surface with $n$ first order branch points over $a_{1}, \cdots, a_{n}$, an $n$th order branch point over $-a_{0}$, and a branch point of order $2 n$ over $w=\infty$. This Riemann surface is the image of the closed $z$-plane by a rational function which we may take to be a polynomial since there is only one point of $\mathcal{F}_{n}$ over $w=\infty$. We normalize this map to correspond to that of the complete surface: 


$$
\begin{gathered}
w=P_{n}(z) \quad \text { of degree } 2 n+1, \\
P_{n}(0)=0 \in S_{1}, \quad P_{n}^{\prime}(0)=1 .
\end{gathered}
$$

The image of the branch point over $w=a_{k}$ is $z=b_{n, k}(k=1,2, \cdots, n)$, $0<b_{n, 1}<\cdots<b_{n, n}$. The image of the $n$th order branch point is $z=-c_{n}$, $c_{n}>0$. The fundamental regions are bounded by curves $C_{n, k}(k=1, \cdots, n)$ through $b_{n, k}$ and by $2 n$ curved rays emanating from $-c_{n}$.

Let $D_{n}$ be the $z$-plane cut along the real axis except for the interval $\left(-c_{n}, b_{n, n}\right)$. Let $\Delta_{n}$ be that region of the $\zeta$-plane containing the origin and bounded by $|\zeta|=R, \Gamma_{n+1}, \Gamma_{-(n+1)}, C_{n+1}$, and the interval $\left(b_{n}, b_{n+1}\right)$. As is readily seen $D_{n}$ and $\Delta_{n}$ correspond to the same portion of $\mathcal{F}$ by the maps $w=P_{n}(z)$ and $w=f(\zeta)$ respectively. The composite function

$$
\zeta=\psi_{n}(z)=\phi\left(P_{n}(z)\right)
$$

maps $D_{n}$ schlichtly onto $\Delta_{n}$, and by (2) and (5)

$$
\psi_{n}(0)=0, \quad \psi_{n}^{\prime}(0)=1 .
$$

The domain $D_{n}$ contains the disc $|z|<\min \left(c_{n}, b_{n, n}\right)$. Applying the Koebe $1 / 4$-theorem to the map of this disc by $\psi_{n}(z)$ we obtain

$$
R>4^{-1} \min \left(c_{n}, b_{n, n}\right) .
$$

Now $P_{n}^{\prime}(z)$ has simple zeros at $z=b_{n, k}$ and an $n$th order zero at $z=-c_{n}$. Consulting (5), we have

$$
\begin{aligned}
P_{n}^{\prime}(z) & =\left(1+z / c_{n}\right)^{n} \prod_{k=1}^{n}\left(1-z / b_{n, k}\right), \\
P_{n}(z) & =\int_{0}^{z} P_{n}^{\prime}(z) d z
\end{aligned}
$$

In particular,

$$
-a_{0}=\int_{0}^{-c_{n}} P_{n}^{\prime}(z) d z, \quad a_{1}=\int_{0}^{b_{n, 1}} P_{n}^{\prime}(z) d z .
$$

Let $b_{n}^{*}$ be defined by

$$
n / b_{n}^{*}=\sum_{i k=1}^{n} 1 / b_{n, k}
$$

Then $b_{n, 1}<b_{n}^{*}<b_{n, n}$. For $-c_{n}<z<b_{n, 1}$ all the factors in the product (9) are positive, and by the comparison between the geometric and arithmetic means

$$
P_{n}^{\prime}(z)<\left(1+z / c_{n}\right)^{n}\left\{\frac{1}{n} \sum_{k=1}^{n}\left(1-z / b_{n, k}\right)\right\}^{n}=\left(1+z / c_{n}\right)^{n}\left(1-z / b_{n}^{*}\right)^{n}
$$


We consider two possibilities:

(1) $b_{n}^{*} \geqq c_{n}$. Then for $-c_{n}<z<0, P_{n}^{\prime}(z)<\left(1+z / c_{n}\right)^{n}\left(1-z / c_{n}\right)^{n}=\left(1-z^{2} / c_{n}^{2}\right)^{n}$ and by (11)

$$
\begin{aligned}
a_{0} & =\int_{-c_{n}}^{0} P_{n}^{\prime}(z) d z<\int_{-c_{n}}^{0}\left(1-z^{2} / c_{n}^{2}\right)^{n} d z=c_{n} \int_{0}^{\pi / 2} \cos ^{2 n+1} t d t \\
& =c_{n} \pi^{1 / 2} \Gamma(n+1) / 2 \Gamma(n+3 / 2) \sim \pi^{1 / 2} c_{n} / 2 n^{1 / 2} .
\end{aligned}
$$

Thus $b_{n}^{*} \geqq c_{n}>A n^{1 / 2}, A>0$.

(2) $c_{n}>b_{n}^{*}$. Then for $0<z<b_{n, 1}, P_{n}^{\prime}(z)<\left(1+z / b_{n}^{*}\right)^{n}\left(1-z / b_{n}^{*}\right)^{n}=\left(1-z^{2} / b_{n}^{* 2}\right)^{n}$ and by (11)

$$
\begin{aligned}
a_{1} & =\int_{0}^{b_{n, 1}} P_{n}^{\prime}(z) d z<\int_{0}^{b_{n, 1}}\left(1-z^{2} / b_{n}^{* 2}\right)^{n} d z<\int_{0}^{b_{n}^{*}}\left(1-z^{2} / b_{n}^{* 2}\right)^{n} d z \\
& =b_{n}^{*} \int_{0}^{\pi / 2} \cos ^{2 n+1} t d t \sim \pi^{1 / 2} b_{n}^{*} / 2 n^{1 / 2} .
\end{aligned}
$$

Thus $c_{n} \geqq b_{n}^{*}>A n^{1 / 2}, A>0$.

In either case, by (12)

$$
c_{n}>A n^{1 / 2}, \quad b_{n, n}>b_{n}^{*}>A n^{1 / 2},
$$

By (8) and (13) $R=\infty$, that is, $\mathcal{F}$ is parabolic.

4. The structure of the entire function $w=f(z)$. The following is a standard theorem (cf. Bieberbach [1, pp. 13-15]) on families of schlicht mappings: let $A_{n}$ be a sequence of schlicht domains in the $z$-plane, all containing the origin. Let $B_{n}$ be a sequence of schlicht domains in the $\zeta$-plane, all containing the origin. Let $\zeta=F_{n}(z)$ map $A_{n}$ schlichtly on to $B_{n}, F_{n}(0)=0, F_{n}^{\prime}(0)=1$. If the sequence $A_{n}$ converges to its kernel $A$ then a necessary and sufficient condition that $F_{n}(z)$ converge uniformly in any closed subset of $A$ is that the $B_{n}$ 's converge to their kernel $B$. The limit function $F(z)$ maps $A$ schlichtly on to $B$.

We have just shown that the sequence of domains $D_{n}$ converges to the punched $z$-plane, and $\Delta_{n}$ converges to the punched $\zeta$-plane. Hence $\psi_{n}(z) \rightarrow F(z)$, $F(0)=0, F^{\prime}(0)=1$, and $\zeta=F(z)$ maps the finite $z$-plane on to the finite $\zeta$-plane. Therefore $F(z)=z$. Thus by (1), (6)

$$
P_{n}(z) \rightarrow f(z), \quad P_{n}^{\prime}(z) \rightarrow f^{\prime}(z)
$$

uniformly for $|z|$ bounded. By Hurwitz' theorem the zeros of $P_{n}^{\prime}(z)$ tend to those of $f^{\prime}(z)$,

$$
\lim _{n \rightarrow \infty} b_{n, k}=b_{k}, \quad k=1,2, \cdots,
$$

and the multiple zero of $P_{n}^{\prime}(z)$ at $z=-c_{n}$ passes out of the picture by (13). Note that (15) is of course not uniform for all $k$.

For $|z|<b_{1} / 2$ and $n>n_{0}, P_{n}^{\prime}(z)$ and $f^{\prime}(z)$ do not vanish and $\log P_{n}^{\prime}(z)$ 
$\rightarrow \log f^{\prime}(z)$, where we take that determination of the logarithm for which $\log P_{n}^{\prime}(0)=\log f^{\prime}(0)=0$. From (9)

(16) $\log P_{n}^{\prime}(z)=\left(n / c_{n}-\sum_{k=1}^{n} 1 / b_{n, k}\right) z-\frac{1}{2}\left(n / c_{n}^{2}+\sum_{k=1}^{n} 1 / b_{n, k}^{2}\right) z^{2}+\cdots$,

and therefore the following limits exist:

$$
\begin{aligned}
\lim _{n \rightarrow \infty}\left(n / c_{n}-\sum_{k=1}^{n} 1 / b_{n, k}\right) & =\sigma_{1}, \\
\lim _{n \rightarrow \infty} \frac{1}{2}\left(n / c_{n}^{2}+\sum_{k=1}^{n} 1 / b_{n, k}^{2}\right) & =\sigma_{2}>0 .
\end{aligned}
$$

All terms in the parenthesis of (18) are positive, hence bounded for all $n$ :

$$
n / c_{n}^{2}<M, \quad \sum_{k=1}^{n} 1 / b_{n, k}^{2}<M
$$

For any fixed $N$, by (15), $2^{-1} \sum_{k=1}^{N} 1 / b_{k}^{2}=\lim _{n \rightarrow \infty} 2^{-1} \sum_{k=1}^{N} 1 / b_{n, k}^{2} \leqq \lim \inf _{n \rightarrow \infty} 2^{-1}$ $\cdot \sum_{k=1}^{n} 1 / b_{n, k}^{2} \leqq \sigma_{2}$, and allowing $N$ to become infinite

$$
\sigma_{3}=\frac{1}{2} \sum_{k=1}^{\infty} 1 / b_{k}^{2} \leqq \sigma_{2} .
$$

Now from (19), (20), and the monotone character of the sequences $b_{k}, b_{n, k}$

$$
c_{n}>A n^{1 / 2}, \quad b_{n, k}>A k^{1 / 2}, \quad b_{k}>A k^{1 / 2}
$$

for some positive constant $A$. For $p \geqq 3$ and any $N$

$$
\begin{aligned}
\underset{n \rightarrow \infty}{\limsup }\left|\sum_{k=1}^{\infty} b_{k}^{-p}-\sum_{k=1}^{n} b_{n, k}^{-p}\right| \leqq \limsup _{n \rightarrow \infty} \sum_{k=1}^{N}\left|b_{k}^{-p}-b_{n, k}^{-p}\right| & \\
& +\sum_{k=N+1}^{\infty} b_{k}^{-p}+\limsup _{n \rightarrow \infty} \sum_{k=N+1}^{n} b_{n, k .}^{-p} .
\end{aligned}
$$

If we use (15) and (21) the first term on the right vanishes and the last two are $O\left(N^{1-p / 2}\right)$; if we allow $N$ to become infinite

$$
\lim _{n \rightarrow \infty} \sum_{k=1}^{n} 1 / b_{n, k}^{p}=\sum_{k=1}^{\infty} 1 / b_{k}^{p}, \quad p \geqq 3 .
$$

If we combine (16), (17), (18), (21), (22)

$$
\log f^{\prime}(z)=\sigma_{1} z-\sigma_{2} z^{2}-\sum_{p=3}^{\infty}\left(z^{p} / p\right) \sum_{k=1}^{\infty} b_{k}^{-p}
$$

By (20) the canonical product 


$$
\Pi(z)=\prod_{k=1}^{\infty}\left\{\left(1-z / b_{k}\right) e^{z / b_{k}}\right\}
$$

converges, and for $|z|$ small

$$
\log \Pi(z)=-\left(z^{2} / 2\right) \sum_{k=1}^{\infty} b_{k}^{-2}-\sum_{p=3}^{\infty}\left(z^{p} / p\right) \sum_{k=1}^{\infty} b_{k}^{-p} .
$$

Comparing this with (23) and (20): $\log f^{\prime}(z)=\sigma_{1} z-\left(\sigma_{2}-\sigma_{3}\right) z^{2}+\log \Pi(z)$ with $\sigma_{2}-\sigma_{3} \geqq 0$. Writing $\sigma_{2}-\sigma_{3}=\alpha, \sigma_{1}=\beta$, we have

$$
f^{\prime}(z)=e^{-\alpha z^{2}+\beta z} \Pi(z)
$$$$
\alpha \geqq 0, \beta \text { real. }
$$

Now $f(z)$ must have a finite asymptotic value along the negative end of the real axis corresponding to the logarithmic branch point of $\mathcal{F}$ over $w=-a_{0}$. Hence the in tegral $\int_{0}^{-\infty} \exp \left(-\alpha x^{2}+\beta x\right) \Pi(x) d x$ must converge. So we must have either $\alpha>0$ or $\beta+\sum_{k=1}^{\infty} 1 / b_{k}>0$. If $\sum 1 / b_{k}$ diverges this condition is met, but as we shall see later (Theorem Ia) this sum may converge.

Summarizing these results we have the following theorem.

THEOREM I. The symmetric gammic surface $\mathcal{F}$ determined by any sequence of real numbers $a_{k}(k=1,2, \cdots), a_{1}>0, a_{2 n \pm 1}>a_{2 n}$, and any $a_{0}>0$ is always parabolic. Furthermore, $\mathcal{F}$ is the (1-1) image of the z-plane by an entire function

$$
\begin{gathered}
w=f(z)=\int_{0}^{z} f^{\prime}(t) d t, \\
f^{\prime}(z)=e^{-\alpha z^{2}+\beta z} \prod_{k=1}^{\infty}\left\{\left(1-z / b_{k}\right) e^{z / b_{k}}\right\}, \\
0<b_{1}<b_{2}<\cdots, \sum b_{k}^{-2}<\infty, \\
\alpha \geqq 0, \quad \beta \text { real, } \max \left\{\alpha, \beta+\sum_{k=1}^{\infty} 1 / b_{k}\right\}>0,
\end{gathered}
$$

the branch point over $w=a_{k}$ corresponding to $z=b_{k}$.

Remark. If the normalization $w(0)=0 \in S_{1}, w^{\prime}(0)=1$, is dropped we obtain: any entire function $w=g(z)$ which maps the $z$-plane $(1-1)$ on to $\mathcal{F}$ is of the form $g(z)=f(p z+q), p, q$ constants, $p \neq 0$.

In a very similar fashion we obtain the following results for the other two classes of surfaces described in $\$ 2$.

THEOREM II. The symmetric semi-cosinic surface $\mathcal{Z}$ is always parabolic; it is the (1-1) image of the z-plane by an entire function

$$
w=f(z)=\int_{0}^{z} f^{\prime}(t) d t,
$$




$$
\begin{gathered}
f^{\prime}(z)=e^{-\delta z} \prod_{k=1}^{\infty}\left(1-z / b_{k}\right), \\
0<b_{1}<b_{2}<\cdots, \sum 1 / b_{k}<\infty, \\
\delta \geqq 0,
\end{gathered}
$$

the branch point over $w=a_{k}$ corresponding to $z=b_{k}$, and $f(0)=0 \in S_{1}$.

THEOREM III. The symmetric cosinic surface $\mathcal{F}$ is always parabolic; it is the (1-1) image of the z-plane by an entire function

$$
\begin{gathered}
w=f(z)=\int_{0}^{z} f^{\prime}(t) d t, \\
f^{\prime}(z)=e^{-\alpha z^{2}+\beta z} \prod_{k=-\infty}\left\{\left(1-z / b_{k}\right) e^{z / b_{k}}\right\}, \\
\cdots<b_{-1}<b_{0}<0<b_{1}<b_{2}<\cdots, \sum b_{k}^{-2}<\infty, \\
\alpha \geqq 0, \quad \beta \text { real, }
\end{gathered}
$$

the branch point over $w=a_{k}$ corresponding to $z=b_{k}$, and $f(0)=0 \in S_{0}$.

Remarks. In Theorems I and III, $\sum 1 / b_{k}$ may or may not converge ( $\$ 5$ will show that both possibilities may occur). If $\sum 1 / b_{k}$ converges then the representation (26b) or (28b) may be simplified. Note that then (26d) and (27d) prevent overlapping between (26b) and (27b).

5. The converse problem. The converses of the preceeding theorems are also true, that is:

Theorem Ia. Any entire function of the form (26abcd) maps the $z$-plane (1-1) onto the symmetric gammic surface with $a_{k}=f\left(b_{k}\right),-a_{0}=\lim _{x \rightarrow-\infty} f(x)$, $x$ real.

Theorem IIa. Any entire function of the form (27abcd) maps the z-plane (1-1) onto the symmetric semi-cosinic surface with $a_{k}=f\left(b_{k}\right)$.

THEOREM IIIa. Any entire function of the form (28abcd) maps the z-plane (1-1) onto the symmetric cosinic surface with $a_{k}=f\left(b_{k}\right)$.

The proofs of these three theorems are similar, and we consider only the first. The essence of the proof is to construct the fundamental regions for $w=f(z)$ by finding the real paths of $f(z)$. Now it is obvious that any $f(z)$ of the form (26) has, among others, the following real paths: the real axis, and one curve $C_{k}$ through each point $z=b_{k}(k=1,2, \cdots)$ symmetric about the real axis. Furthermore, no two real paths intersect except at the critical points $b_{k}$. But it is not obvious that $f(z)$ also has the appropriate real paths $\Gamma_{ \pm k}$ described in $\S 3$, nor is it immediate that there are no more real paths between the $C_{k}$ 's. The easiest procedure is to use a sequence of polynomials which 
approximate $f(z)$ and which correspond to easily constructed surfaces.

Let

$$
\beta_{n}=\beta+\sum_{k=1}^{n} 1 / b_{k}
$$

For any sequence of positive integers $\lambda_{n}$ which increase rapidly enough the polynomials

$$
\begin{aligned}
Q_{n}(z) & =\int_{0}^{z} Q_{n}^{\prime}(t) d t, \\
Q_{n}^{\prime}(z) & =\left(1-\alpha z^{2} / \lambda_{n}\right)^{\lambda_{n}}\left(1+\beta_{n} z / \lambda_{n}\right)^{\lambda_{n}} \prod_{k=1}^{n}\left(1-z / b_{k}\right)
\end{aligned}
$$

approximate $f(z)$ :

$$
\lim _{n \rightarrow \infty} Q_{n}^{\prime}(z)=f^{\prime}(z), \quad \lim _{n \rightarrow \infty} Q_{n}(z)=f(z)
$$

uniformly for $|z|$ bounded. This depends on

$$
\lim _{N \rightarrow \infty}(1+z / N)^{N}=e^{z}, \quad \text { uniformly for }|z| \leqq R .
$$

Thus

$$
\lim _{n \rightarrow \infty}\left(1-\alpha z^{2} / \lambda_{n}\right)^{\lambda_{n}}=e^{-\alpha z^{2}}
$$

Also

$$
\begin{aligned}
\mid\left(1+\beta_{n} z / \lambda_{n}\right)^{\lambda_{n}} \prod_{k=1}^{n} & \left(1-z / b_{k}\right)-e^{\beta z} \prod_{k=1}^{\infty}\left\{\left(1-z / b_{k}\right) e^{z / b_{k}}\right\} \mid \\
\leqq & \left|\left(1+\beta_{n} z / \lambda_{n}\right)^{\lambda_{n}}-e^{\beta_{n} z}\right| \cdot\left|\prod_{k=1}^{n}\left(1-z / b_{k}\right)\right| \\
& \quad+\left|e^{\beta_{n} z} \prod_{k=1}^{n}\left(1-z / b_{k}\right)-e^{\beta z} \prod_{k=1}^{\infty}\left\{\left(1-z / b_{k}\right) e^{z / b_{k}}\right\}\right| .
\end{aligned}
$$

The last term on the right of (35) tends to zero, uniformly for $|z| \leqq R$, by the nature of an infinite product. If we choose $\lambda_{n}$ so that, by virtue of (33) $\left|\left(1+\beta_{n} z / \lambda_{n}\right)^{\lambda_{n}}-e^{\beta_{n} z}\right|<\left[n \prod_{k=1}^{n}\left(1+n / b_{k}\right)\right]^{-1}$ for $|z| \leqq n$, then the left-hand member of (35) tends to zero uniformly for $|z|$ bounded. Combining this with (34) we have the first part of (32); the second follows immediately.

We shall now construct the Riemann surface of the function $z=\phi(w)$, inverse to $w=f(z)$, for the case $\alpha>0$. The general idea is that the real paths of $Q_{n}(z)$ tend to those of $f(z)$. Increasing the terms in the sequence $\lambda_{n}$ does not affect (32), so we shall assume that $\lambda_{n} /\left|\beta_{n}\right|>\left(\lambda_{n} / \alpha\right)^{1 / 2}>b_{n}$. By (31) it is readily seen that the real paths of $Q_{n}(z)$ are as follows: (1) the real axis; (2) curves 
$C_{n, k}(k=1, \cdots, n)$ through $b_{k}$; (3) $2 \lambda_{n}$ curved rays $\Gamma_{n, \pm k}\left(k=1, \cdots, \lambda_{n}\right)$ emanating from $z=-\left(\lambda_{n} / \alpha\right)^{1 / 2}$, those in the upper half-plane being $\Gamma_{n, 1}, \Gamma_{n, 2}$, $\cdots, \Gamma_{n, \lambda_{n}}$ in counterclockwise order about their origin, and $\Gamma_{n,-k}$ being the reflection of $\Gamma_{n, k}$ in the real axis; (4) $2 \lambda_{n}$ curved rays $\Gamma^{\prime}$ emanating from $z=\left(\lambda_{n} / \alpha\right)^{1 / 2}$; and (5) $2 \lambda_{n}$ curved rays $\Gamma^{\prime \prime}$ emanating from $z=-\lambda_{n} / \beta_{n}$. This whole scheme is symmetric about the real axis.

The rays $\Gamma^{\prime}$ and $\Gamma^{\prime \prime}$ disappear in the limit, for if not, some circle $|z| \leqq R$ would contain sections of the limiting curves of all the $C_{n, k}$ and $\Gamma_{n, k}$ (since $\left.\lambda_{n} /\left|\beta_{n}\right|>\left(\lambda_{n} / \alpha\right)^{1 / 2}>b_{n}\right)$, and $f(z)$ would possess sections of infinitely many different real paths in $|z| \leqq R$, which is impossible for an entire function.

The curves $C_{n, k}$ will tend to limiting curves $C_{k}$. They cannot pass out of the picture since $C_{n, k}$ contains the fixed point $z=b_{k}$ for all $n$, but it is conceivable that $C_{k}$ might consist of several pieces, all "ends" being at $z=\infty$. The strip $D_{n, k}$, bounded by $C_{n, k}$ and $C_{n, k+1}$, is mapped by $Q_{n}(z)$ onto a w-plane, $\Delta_{n, k}$, slit along the real axis except for the segment $\left(Q_{n}\left(b_{k}\right), Q_{n}\left(b_{k+1}\right)\right)$. The sequence $\Delta_{n, k}$ converges to its kernel $\Delta_{k}$, the w-plane slit along the real axis except for the segment $\left(a_{k}, a_{k+1}\right)=\left(f\left(b_{k}\right), f\left(b_{k+1}\right)\right)$. Applying the theorem stated at the beginning of $\S 4$ to the sequence of functions $Q_{n}^{-1}(w)$ (the condition $F_{n}(0)=0, F_{n}^{\prime}(0)=1$, is replaced by $Q_{n}^{-1}\left(\left(a_{k}+a_{k+1}\right) / 2\right) \rightarrow A, Q_{n}^{-1}\left(\left(a_{k}+a_{k+1}\right) / 2\right)$ $\rightarrow B \neq 0)$ we see: $w=f(z)$ maps $D_{k}$, the kernel of the sequence $D_{n, k}$, onto $\Delta_{k}$. Thus $D_{k}$ is simply-connected. Furthermore $C_{k}$ is all in one piece, for if we apply similar considerations to the map of the part of $D_{n, k-1}+D_{n, k}$ in the upper half-plane, $f(z)$ is analytic at every point of $C_{k}$ except one at infinity, that is, $C_{k}$ is one connected curve and the regions $D_{1}, D_{2}, \cdots$, fill out the section of the $z$-plane bounded by $C_{1}$ and not containing $z=0$. Comparing this with $\S 3$, this part of the $z$-plane is mapped onto the sheets $S_{2}, S_{3}, \cdots$, of the "semicosinic end" of a gammic surface.

The logarithmic ends are obtained in a similar fashion: the region of the upper half-plane bounded by $C_{n, 1}, \Gamma_{n, 2}$, the real axis, and containing $\Gamma_{n, 1}$ is mapped by $Q_{n}(z)$ onto a plane slit along the real axis from $+\infty$ to $Q_{n}\left(-\left(\lambda_{n} / \alpha\right)^{1 / 2}\right), \Gamma_{n, 1}$ mapping into the remainder of the real axis. Applying the same theorem to this sequence of maps, there are two possibilities: (1) $\Gamma_{n .1}$ (and hence all $\Gamma_{n, k}$ ) disappear in the limit and the (appropriate) region bounded by the real axis and $C_{1}$ is mapped by $w=f(z)$ onto an upper halfplane. This is impossible, for this would imply that $\lim _{x \rightarrow-\infty} f(x)=-\infty$ ( $x$ real), whereas equations (26) imply a finite asymptotic value $=-a_{0}$ along the negative real axis. Hence we have (2), the curves $\Gamma_{1}$ and $\Gamma_{2}$, limits of $\Gamma_{n, 1}$ and $\Gamma_{n, 2}$, actually exist; the region bounded by $\Gamma_{2}$, the real axis, and $C_{1}$ is mapped by $w=f(z)$ onto a plane slit along $\left(-a_{0},+\infty\right)$. The curve $\Gamma_{1}$ is mapped onto $\left(-\infty,-a_{0}\right)$ and hence $\Gamma_{1}$ is all in one piece. Repeating this argument for the various regions between $\Gamma_{n, k}$ and $\Gamma_{n, k+2}$, we see that $f(z)$ has an infinite sequence of real paths $\Gamma_{k}$ as described in $\S 3$, and the associated strips map into the half-sheets of a logarithmic end with singularities over $w=-a_{0}$ and 
$w=\infty$. Since $f(z)$ is symmetric in the real axis we obtain the other logarithmic end, which completes the proof of Theorem Ia, for $\alpha>0$. For $\alpha=0$ the procedure is virtually the same except that the paths $\Gamma_{k}$ arise from the multiple zero of $Q_{n}^{\prime}(z)$ at $z=-\lambda_{n} / \beta_{n}$ (less than 0 for $n$ sufficiently large by (26d)).

6. Some properties of the fundamental regions. The sequence of polynomials $Q_{n}(z)$ may be used to derive properties of the real paths of $w=f(z)$ other than the topological and symmetric properties mentioned in $\$ 3$.

First it may be shown that the line $y=y_{0}>0(z=x+i y)$ intersects all the real paths of $Q_{n}(z)$ which lie in the upper half-plane exactly once. These intersections correspond to the roots of the equation

$$
J Q_{n}\left(x+i y_{0}\right)=0
$$

which, for $\alpha>0$, is of degree $3 \lambda_{n}+n$ in $x$. Considering the fact that the real paths of a polynomial divide the angle at $z=\infty$ equally, the line $y=y_{0}$ must intersect each of the following at least once: $C_{n, 1}, \cdots, C_{n, n} ; \Gamma_{n, 1}, \cdots, \Gamma_{n, \lambda_{n}}$; $\lambda_{n}$ of the $\Gamma^{\prime}$, and $\lambda_{n}$ of the $\Gamma^{\prime \prime}$. But this makes up the precise degree of (36), so there is exactly one simple intersection of the line with each curve mentioned. Since the roots of (36) are real and simple, the roots of

$$
\partial J Q_{n}\left(x+i y_{0}\right) / \partial x=0
$$

are real, simple, and alternate with those of $(3 \dot{6})$. That these facts still obtain in the limit may be proved in various ways, either elementary or by use of Hurwitz' theorem. The roots of

$$
\exists f\left(x+i y_{0}\right)=0,
$$$$
y_{0} \neq 0,
$$

are simple and alternate with the (simple) roots of

$$
\partial \Im f\left(x+i y_{0}\right) / \partial x=0 .
$$

For $\alpha=0$ this is proved in a similar manner. This result does not state that the line $y=y_{0}$ actually intersects $C_{k}$ and $\Gamma_{k}$ for all values of $y_{0}$ and $k$; some of these intersections might disappear in the limit, for example $\Gamma_{1}$ might have a horizontal asymptote $y=y_{1}>0$. We consider this question now. Equation (39) is equivalent to $J f^{\prime}\left(x+i y_{0}\right)=0$, or

$$
\Theta=\arg f^{\prime}\left(x+i y_{0}\right)=-k \pi,
$$$$
k=0, \pm 1, \pm 2, \cdots
$$

Consulting (26b), we have

$$
\Theta=-2 \alpha x y+\beta y+\sum_{k=1}^{\infty}\left\{\frac{y}{b_{k}}-\tan ^{-1} \frac{y}{b_{k}-x}\right\}
$$

where we choose $0<\tan ^{-1}\left(y /\left(b_{k}-x\right)\right)<\pi$. With this determination the sum in (41) is convergent, and $\Theta$ is a continuous function of both $x$ and $y$ for all $x$ and for $y>0$. Differentiating (41), we have 


$$
\frac{\partial \Theta}{\partial x}=-2 \alpha y-\sum_{k=1}^{\infty} y /\left[y^{2}+\left(b_{k}-x\right)^{2}\right] .
$$

Thus $\Theta$ decreases as we move to the right along $y=y_{0}$. Since $\Theta$ is continuous, the value of $k$ in (40) associated with the intersection between two given $C$ or $\Gamma$ curves is a constant. The particular value of this constant may be determined by setting $x=\left(b_{k}+b_{k+1}\right) / 2$ in (41) and letting $y \rightarrow 0$ :

$$
\lim _{y \rightarrow 0} \Theta\left(\left(b_{k}+b_{k+1}\right) / 2, y\right)=-k \pi \text {. }
$$

Let the root of $\Theta(x, y)=-k \pi$ be $x=\xi_{k}(y)$. Let the equation of the curve $C_{k}$ be $x=\chi_{k}(y)$ and the equation of $\Gamma_{k}$ be $x=\chi_{-k}(y)$. Then we have

$$
\begin{aligned}
\xi_{k-1}(y) & <\chi_{k}(y)<\xi_{k}(y), & k & =1,2, \cdots, \\
\xi_{-k}(y) & <\chi_{-k}(y)<\xi_{-k+1}(y), & k & =1,2, \cdots .
\end{aligned}
$$

There is one immediate fact to be drawn from (42) if $\alpha>0$, for then

$$
\left|\Theta\left(x_{2}, y\right)-\Theta\left(x_{1}, y\right)\right|=\int_{x_{1}}^{x_{2}}\left|\frac{\partial \Theta}{\partial x}\right| d x>2 \alpha y\left|x_{2}-x_{1}\right| .
$$

Therefore $0<\xi_{k-1}(y)-\xi_{k}(y)<\pi / 2 \alpha y$, and by (43),

$$
\begin{array}{ll}
0<\chi_{k+1}(y)-\chi_{k}(y)<\pi / \alpha y, & k=1,2, \cdots, \\
0<\chi_{1}(y)-\chi_{-1}(y)<\pi / \alpha y, & \\
0<\chi_{-k}(y)-\chi_{-k-1}(y)<\pi / \alpha y, & k=1,2, \cdots .
\end{array}
$$

In this case, $\alpha>0$, we see that the real paths have no horizontal asymptotes, and the horizontal width of all strips decreases uniformly as $1 / y$.

Now for $x<0, \tan ^{-1}\left(y /\left(b_{k}-x\right)\right)<y /\left(b_{k}-x\right)<y / b_{k}$, and since the sum in (41) is a monotone function of $x$ we have for any $N$

$$
\lim _{x \rightarrow-\infty} \sum_{k=1}^{\infty}\left\{\frac{y}{b_{k}}-\tan ^{-1} \frac{y}{b_{k}-x}\right\} \geqq \lim _{x \rightarrow-\infty} \sum_{k=1}^{N}\left\{\frac{y}{b_{k}}-\tan ^{-1} \frac{y}{b_{k}-x}\right\}=\sum_{k=1}^{N} \frac{y}{b_{k}} \text {. }
$$

Also

$$
\lim _{x \rightarrow-\infty} \sum_{k=1}^{\infty}\left\{\frac{y}{b_{k}}-\tan ^{-1} \frac{y}{b_{k}-x}\right\} \leqq \sum_{k=1}^{\infty} \frac{y}{b_{k}} .
$$

Thus this limit is $y \sum_{k=1}^{\infty} 1 / b_{k}$. If $\alpha=0$, then

$$
\lim _{x \rightarrow-\infty} \Theta(x, y)=y\left(\beta+\sum_{k=1}^{\infty} 1 / b_{k}\right) .
$$

Thus if $\sum 1 / b_{k}$ diverges all curves $\Gamma$ are intersected by any horizontal line, but if $\sum_{k=1}^{\infty} 1 / b_{k}=t<\infty$ then $y=y_{0}$ intersects $\Gamma_{k}$ for $y_{0}>k \pi /(\beta+t)$ but does 
not in tersect $\Gamma_{k}$ for $y_{0}<(k-1) \pi /(\beta+t)$.

On the other side of the picture, $\Theta$ is a monotone function of $x$ and so $\lim _{x \rightarrow+\infty} \Theta\left(x, y_{0}\right)$ exists and the value determines roughly which curves $C_{k}$ are intersected by $y=y_{0}$. Here we consider only $\alpha=0$, since we know the answer by (44) when $\alpha>0$.

For $b_{n}<x<b_{n+1}, b_{n}>y$, we have from (42)

$$
\begin{gathered}
\frac{\partial \Theta}{\partial x}<-\sum_{k=1}^{n}\left(y /\left(y^{2}+x^{2}\right)-\sum_{k=n+1}^{\infty} y /\left(y^{2}+b_{k}^{2}\right)\right. \\
<-\sum_{k=1}^{n} y /\left(x^{2}+x^{2}\right)=-n y / 2 x^{2}, \\
\Theta\left(b_{n}, y\right)-\Theta\left(b_{n+1}, y\right)=\int_{b_{n}}^{b_{n}+1}-\frac{\partial \Theta}{\partial x} d x>\frac{n y}{2}\left(\frac{1}{b_{n}}-\frac{1}{b_{n+1}}\right) .
\end{gathered}
$$

Adding these inequalities for all $n \geqq m$, where $b_{m}>y$ :

$$
\begin{aligned}
\Theta\left(b_{m}, y\right)-\lim _{x \rightarrow \infty} \Theta(x, y) & >\frac{y}{2} \sum_{n=m}^{\infty}\left(\frac{n}{b_{n}}-\frac{n}{b_{n+1}}\right) \\
& =\frac{y}{2}\left(m / b_{m}+\sum_{n=m+1}^{\infty} 1 / b_{n}\right) .
\end{aligned}
$$

Therefore if $\sum 1 / b_{n}$ diverges, $\lim _{x \rightarrow \infty} \Theta(x, y)=-\infty$ and $C_{n}$ is intersected by every horizontal line.

If $\sum 1 / b_{n}$ converges we proceed as follows. From (41), $\alpha=0$,

$$
\Theta=\beta y+y \sum_{n=1}^{\infty} 1 / b_{n}-\sum_{n=1}^{\infty} \tan ^{-1} \frac{y}{b_{n}-x} .
$$

Then since $\tan ^{-1}\left(y\left(b_{n}-x\right)\right)>0$ we have for $x>b_{m}, \Theta<\beta y+y \sum_{n=1}^{\infty} 1 / b_{n}$ $-\sum_{n=1}^{m} \pi / 2=A y-m \pi / 2$ and $\lim _{x \rightarrow \infty} \Theta(x, y)=-\infty$, and again, $C_{k}$ is intersected by every horizontal line.

Sharper results on the course of the real paths may obviously be obtained using the relation between (39) and (40), especially when a reasonably regular behaviour is assumed for the sequence $b_{n}$.

The problem of the relation between the numbers $\alpha, \beta, b_{n}$ and the branch points, $a_{n}$, of the Riemann surface is not simple. The following however may easily be established: if $\alpha>0$ then

$$
\sum_{k=n}^{\infty}\left|a_{k+1}-a_{k}\right|=O\left(e^{-(\alpha-\epsilon) b_{n}^{2}}\right)=o\left(e^{-\gamma n}\right)
$$

for any positive $\gamma$. For the canonical product in (26) is $O\left(e^{e r^{2}}\right)$ for any $\epsilon>0$. Thus $\sum_{k=n}^{\infty}\left|a_{k+1}-a_{k}\right|=\int_{b_{n}}^{\infty}\left|f^{\prime}(z)\right| d z=O\left(e^{-(\alpha-\epsilon) b_{n}^{2}}\right)$, which is the first part of (47). The rest follows since $b_{n}^{2} / n \rightarrow \infty$. 
Results similar to the above may be stated for the other two classes of surfaces. The semi-cosinic fundamental regions, associated with (27), have the following properties:

(1) The real paths of $w=f(z)$ consist of the real axis and one curve $C_{n}$ through each critical point $b_{n}$, symmetric about the real axis.

(2) As the point $P$ travels along the upper half of $C_{n}$ from $b_{n}$ to infinity its ordinate increases monotonically.

(3) Each $C_{n}$ eventually enters the half-plane $R(z)<b_{1}$. The upper half of $C_{n}$ has a finite negative slope at every point of this half-plane. $C_{n}$ has precisely one simple intersection with the line $R(z)=x_{0}<b_{1}$ in the upper half-plane. Let this point be $\left(x_{0}, y_{n}\left(x_{0}\right)\right)$.

(4) The values $y_{n}\left(x_{0}\right)$ alternate with the simple roots of $\arg f^{\prime}\left(x_{0}-i y\right)$ $=(2 k-1) \pi / 2$. If $\arg f^{\prime}\left(x_{0}\right)=0$ and $\arg f^{\prime}\left(x_{0}-i \eta_{k}\right)=(2 k-1) \pi / 2$, then $0<\eta_{1}$ $<y_{1}<\eta_{2}<y_{2}<\ldots$.

(5) Each $C_{n}$ is simply visible from any point $z<b_{1}$ of the real axis.

(6) $C_{k}$ lies entirely in the half-plane $R(z)<b_{2 k-1}$.

(7) If $\delta>0$, then $\lim _{x \rightarrow \infty} f(x)=a \neq \infty, x$ real, and

$$
\sum_{k=n}^{\infty}\left|a_{k+1}-a_{k}\right|<A(\epsilon) e^{-(\delta-\epsilon) b_{n}} .
$$

If $\delta=0$ and $\lim _{x \rightarrow \infty} f(x)=a \neq \infty$, then there exists an infinite subsequence of indices $n$ for which $\left|a_{n}-a\right|>K(\epsilon) e^{-\epsilon \operatorname{con}+1}$.

The cosinic fundamental regions associated with (28) have the following properties:

(1) The real paths of $w=f(z)$ consist of the real axis and a curve $C_{n}$ through each critical point $b_{n}$, symmetric about the real axis.

(2) The line $y=y_{0}$ intersects each $C_{n}$ exactly once.

(3) If $\alpha>0$, the horizontal width of the strip between $C_{n}$ and $C_{n+1}$ is $O(1 / y)$ uniformly for all $n$.

(4) If $\alpha>0$, then

$$
\sum_{k=n}^{\infty}\left|a_{k+1}-a_{k}\right|=O\left(e^{-(\alpha-\epsilon) b_{n}^{2}}\right), \quad \sum_{k=-n}^{\infty}\left|a_{k+1}-a_{k}\right|=O\left(e^{-(\alpha-\epsilon) b^{2}-n}\right) .
$$

7. Remarks. As is immediately seen upon examination of the proofs of both the direct and converse theorems, the restriction $a_{2 n_{ \pm}}>a_{2 n}$, or its equivalent $b_{n+1}>b_{n}$, may be replaced by the weaker $b_{n+1} \geqq b_{n}$; that is, a group of $p$ consecutive first-order branch points may be collapsed into a single branch point of order $p$, with no essential change in the statement of the theorem involved.

Concerning the product representations for $w=f(z)$ we may say:

THEOREM Ib. If $\mathcal{F}$ is a symmetric gammic surface with $a_{2 n+1}>0, a_{2 n}<0$, then $w=f(z)$ (cf. (26)) is of the form 


$$
f(z)=z e^{-\gamma z^{2}+\delta z} \prod_{n=1}^{\infty}\left\{\left(1-z / c_{n}\right) e^{z / c_{n}}\right\}
$$

with $\gamma \geqq 0, \delta$ real, $0<c_{1}<c_{2}<\cdots$, and $\max \left(\gamma, \delta+\sum_{n=1}^{\infty} 1 / c_{n}\right)>0$. And conversely, an entire function of this form maps the z-plane onto a symmetric gammic surface $\mathcal{F}$ with $a_{2 n+1}>0, a_{2 n}<0$.

The proof of this theorem is obvious. Similar results obtain for the other two classes of surfaces.

This enables us to conclude at once that the Riemann surface corresponding to the entire function $w=z^{-n} J_{n}(z)$, where $J_{n}(z)$ is the Bessel function of order $n>-1$, is a symmetric cosinic surface. For the zeros of $w(z)$ are real and (cf. Watson [1, pp. 482 and 498]) $w \Gamma(n+1) 2^{n}=\prod_{k=-\infty}^{\infty}\left\{\left(1-z / c_{k}\right) e^{z / c_{k}}\right\}$. The Riemann surfaces connected with the Bessel functions have been considered by Lense [2].

When the condition sgn $a_{n}=(-)^{n-1}$ is not fulfilled $f(z)$ has certain complex zeros occurring in conjugate pairs, and the story is not so simple; for example, if $f(z)=z\left(1+z^{2} / 3\right) \prod_{n=1}^{\infty}\left(1-z / b_{n}\right), b_{n}>0, f^{\prime}(z)$ will have zeros in the vicinity of $\pm i$ if the sequence $b_{n}$ is chosen large enough, since the derivative of $z\left(1+z^{2} / 3\right)$ has zeros at $\pm i$, and hence $f(z)$ will not correspond to a symmetric semi-cosinic surface.

A comparison of Theorems I and III suggests that it should be possible to replace a pair of logarithmic ends by a symmetric "semi-cosinic end" without altering the type of the Riemann surface. That this is true in fairly general situations will be shown in a later paper.

\section{REFERENCES}

\section{BIEBERBACH}

1. Lehrbuch der Funktionentheorie, 2d ed., vol. 2, Leipzig, 1931.

I. GINZEL

1. Die konforme Abbildung durch die Gammafunktion, Acta Math. vol. 56 (1931).

F. IVERSEN

1. Recherches sur les fonctions inverses des fonctions meromorphes. Thesis, Helsingfors, 1914. J. LENSE

1. Über die konforme Abbildung durch die Gammafunktion, Akademie der Wissenschaften, Munich, Mathematisch-Physikalische Classe, Sitzungsberichte (1928).

2. Über die konforme Abbildung durch die Besselfunktionen, Akademie der Wissenschaften, Munich, Mathematisch-Physikalische Classe, Sitzungsberichte (1932).

R. Nevanlinna

1. Eindeutige Analytische Funktionen, Berlin, 1936.

F. E. UlRICH

1. The problem of type for a certain class of Riemann surfaces, Duke Math. J. vol. 5 (1939).

G. N. Watson

1. A treatise on the theory of Bessel functions, 2d ed., New York, 1944.

The Rice Institute,

Houston, Tex. 